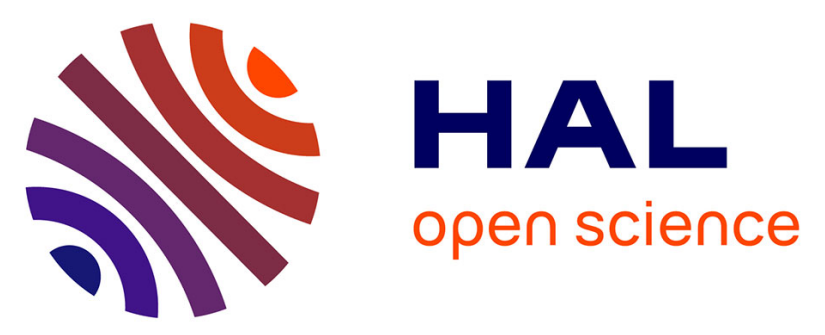

\title{
Observer-Based Current controller for virtual synchronous generator in presence of unknown and unpredictable loads
}

\author{
Audrey Moulichon, Mazen Alamir, Vincent Debusschere, Lauric Garbuio, \\ Mustapha Amine Rahmani, Miao-Xin Wang, Nouredine Hadjsaid
}

\section{To cite this version:}

Audrey Moulichon, Mazen Alamir, Vincent Debusschere, Lauric Garbuio, Mustapha Amine Rahmani, et al.. Observer-Based Current controller for virtual synchronous generator in presence of unknown and unpredictable loads. IEEE Transactions on Power Electronics, 2020, 36 (2), pp.1708-1716. 10.1109/TPEL.2020.3010085 . hal-03015963

\section{HAL Id: hal-03015963 \\ https://hal.science/hal-03015963}

Submitted on 20 Nov 2020

HAL is a multi-disciplinary open access archive for the deposit and dissemination of scientific research documents, whether they are published or not. The documents may come from teaching and research institutions in France or abroad, or from public or private research centers.
L'archive ouverte pluridisciplinaire HAL, est destinée au dépôt et à la diffusion de documents scientifiques de niveau recherche, publiés ou non, émanant des établissements d'enseignement et de recherche français ou étrangers, des laboratoires publics ou privés. 


\title{
Observer-Based Current Controller for Virtual Synchronous Generator in Presence of Unknown and Unpredictable Loads
}

\author{
Audrey Moulichon ${ }^{1,2,3}$, Student Member, IEEE, Mazen Alamir ${ }^{2}$, Vincent Debusschere ${ }^{1}$, Member, IEEE, \\ Lauric Garbuio $^{1}$, Member, IEEE, Mustapha Amine Rahmani ${ }^{3}$, Miao-Xin Wang ${ }^{3}$, \\ and Nouredine Hadjsaid ${ }^{1}$, Life Fellow, IEEE
}

\begin{abstract}
This paper proposes an observer-based current controller for virtual synchronous generators (VSG) in the presence of unmeasured and unpredictable loads. First, the considered models are described, notably the selected VSG model, as well as the grid-connected inverter. Then, the proposed controller is detailed which consists in a linear quadratic regulator incorporating an integrator and a state observer. Finally, experimental results validate the capacity of the VSG solution, based on the presented controller, to supply unknown and unpredictable loads represented as nonlinear and unbalanced loads, as well as survive short-circuits and operate in parallel with other power sources. This extensive set of tests proves the relevance of such controller in the wake of the industrialization of the proposed VSG solution, as well as the necessity to incorporate a nonsimplified synchronous machine model and an advance, reactive controller.
\end{abstract}

Index Terms-Grid Forming Inverters, Microgrids, Power Generation, Renewable Energy, State Observer, State-Space Model, Synchronous Machine, Synchronverter, Virtual Synchronous Generator.

\section{INTRODUCTION}

$\mathbf{T}$ HE traditional distributed energy resources (DER) installed to supply microgrids, diesel generator sets, are becoming less attractive because of the increasing cost of fuel and its associated environmental impact. They are being gradually supplanted by renewable energy sources (RES). However, since this new power sources are intermittent, they could create in time major stability issues. Indeed, the insertion of RES as main supplying power source decreases the microgrid's inertia and its capacity to remain stable after a harsh load impact [1]. A good candidate solution, bringing some answers to those issues while still allowing the increased integration of RES, is the virtual synchronous generator (VSG).

${ }^{1}$ A. Moulichon, V. Debusschere, L. Garbuio and N. Hadjsaid are with Univ. Grenoble Alpes, CNRS, Grenoble INP*, G2Elab, F-38000 Grenoble, France. lauric.garbuio@g2elab.grenoble-inp.fr, vincent.debusschere@grenoble-inp.fr, nouredine.hadjsaid@g2elab.grenoble-inp.fr

2 A. Moulichon and M. Alamir are with Univ. Grenoble Alpes, CNRS, Grenoble INP*, Gipsa-lab, F-38000 Grenoble, France. mazen.alamir@gipsalab.grenoble-inp.fr

${ }^{3}$ A. Moulichon, M.A. Rahmani and M.-X. Wang are with the Power Conversion department, SCHNEIDER ELECTRIC INDUSTRIES, 38000 Grenoble, France. audrey.moulichon@se.com, mustapha.rahmani@se.com, miaoxin.wang@se.com.

*Institute of Engineering Univ. Grenoble Alpes.

Manuscript received Month XX, 2019; revised Month XX, 2020
Nomenclature

\begin{tabular}{|c|c|}
\hline Symbol & Definition \\
\hline$\psi_{d}$ and $\psi_{q}$ & Machine $d q$ stator flux linkages \\
\hline$\psi_{1 d}, \psi_{1 q}$ and $\psi_{f d}$ & Machine $d q$ rotor flux linkages \\
\hline$\omega_{r}$ and $\omega_{\text {base }}$ & $\begin{array}{l}\text { Machine rotor electrical angular velocity and its } \\
\text { base value, } \omega_{r}=\omega_{\text {rotor }} / \omega_{\text {base }}\end{array}$ \\
\hline$i_{d}$ and $i_{q}$ & Machine $d q$ stator output current \\
\hline$e_{f d}$ & Machine d-axis excitation voltage \\
\hline$i_{1 d}, i_{1 q}$ and $i_{f d}$ & Machine $d q$ damper currents and field current \\
\hline$R_{a}$ & Machine stator line (armature) resistance \\
\hline$L_{a d}$ and $L_{a q}$ & Machine $d q$ stator-rotor mutual inductance \\
\hline$L_{l}$ & Machine stator leakage inductance \\
\hline$L_{1 d}, L_{1 q}$ and $L_{f d}$ & Machine rotor circuit leakage inductances \\
\hline$L_{d}^{\prime}, L_{d}^{\prime \prime}$ and $L_{q}^{\prime \prime}$ & Machine d-axis transient, $d q$ subtransient reactance \\
\hline$T_{d o}^{\prime}$ and $T_{d o}^{\prime \prime}$ & $\begin{array}{l}\text { Machine d-axis transient and subtransient open- } \\
\text { circuit time }\end{array}$ \\
\hline$T_{q o}^{\prime \prime}$ & Machine q-axis subtransient open-circuit time \\
\hline$V_{D C}$ & Inverter input DC voltage \\
\hline$\alpha_{d}$ and $\alpha_{q}$ & $d q$ inverter output duty ratio \\
\hline$V_{i}, V_{i}^{d}$ and $V_{i}^{q}$ & $\begin{array}{l}\text { Unifilar and } d q \text { inverter voltage considering: } \\
V_{i}=\alpha \cdot \frac{V_{D C}}{2}\end{array}$ \\
\hline$E, e^{d}$ and $e^{q}$ & Single-line and $d q$ filter voltage \\
\hline$V_{g}, V_{g}^{d}$ and $V_{g}^{q}$ & Single-line and $d q$ grid voltage \\
\hline$i_{L}, i_{L}^{d}$ and $i_{L}^{q}$ & Single-line and $d q$ output inverter current \\
\hline$i_{g}, i_{g}^{d}$ and $i_{g}^{q}$ & Single-line and $d q$ grid inverter current \\
\hline$L_{L}$ and $R_{L}$ & Inverter's inductance and resistor \\
\hline$L_{g}$ and $R_{g}$ & Grid's inductance and resistor \\
\hline$C_{f}$ and $R_{f}$ & $\begin{array}{l}\text { Capacitive filter's inductance and resistor } \\
\text { Notation }\end{array}$ \\
\hline $\mathbb{I}_{n}$ & Eye matrix of size $n$ \\
\hline $\mathbb{O}_{i, j}$ & Full zeros matrix of $i$ rows and $j$ colons \\
\hline$M^{t}$ & Transposed matrix of $M$ \\
\hline$M^{s}$ & Discrete state space model of $M$ \\
\hline$\hat{M}$ & Observed vectors or system \\
\hline$M^{+}$ & Next step state-space value of $M$ \\
\hline$M^{*}$ & Reference of the state vector $M$ \\
\hline $\bar{M}$ & $\begin{array}{c}\text { Extended state vector of } M \\
\text { Acronym }\end{array}$ \\
\hline IBG & Inverter-based Generation \\
\hline DER & Distributed Energy Resource \\
\hline LQR & Lenear Quadratic Regulator \\
\hline RES & Renewable Energy Source \\
\hline $\mathrm{SM}$ & Synchronous Machine \\
\hline VSG & Virtual Synchronous Machine \\
\hline
\end{tabular}

The first works on VSG solutions for inverters used a complete synchronous machine (SM) model with the flux linkages and damper windings effects [2], [3]. Other SM models were also used as reference for the VSG, for instance, a simplified machine model without flux linkages nor dampers 
[4], [5] or an algebraic model [6], [7]. Various projects have shown the advantages of VSG for the insertion of RES in microgrids [8], [9].

The droop control possibly implemented in VSG is also extensively studied to improve the necessary feedback actions that prove necessary to guarantee an acceptable behavior through current, voltage and frequency control. Indeed, depending of the controller, the voltage and frequency performances can be improved by modifying the droop during load variations [10]-[12] and also self-synchronization with other power sources [13]. In addition, as the SM model is virtual, the self-tuning of its parameters is also under investigation [14], [15]. One of the most frequently used solution is the multiloop control architecture that implements the three different regulations, namely on current, voltage and frequency [16]. Other solutions have also been implemented such as traditional ones based on a proportional integrator (PI) corrector with the addition of a resonant [17], a robust $\mathrm{H} \infty$ method [18] or a fuzzy controller [19] for instance.

The controllers are developed both to increase the performances of the VSG and to minimize the creation of oscillations at the output of the inverter [20]. The oscillations are caused partly by resonances occurring after the grid's frequency which then destabilize the controlled system [3]. A state-space methodology has been used in [21] to develop an optimized controller avoiding the creation of oscillations. However, it is restrictive as the entire configuration of the microgrid must be implemented in the controller. It needs to be modified each time the microgrid evolves.

To the best of our knowledge, the use of an observer has not been considered in the controller of a VSG. The solution proposed in this paper is a combination of a controller based on the state-space model of the SM (adapted to an actual gridconnected inverter) and an observer to reconstruct the load's characteristics.

This paper is organized in three parts: First, the models used to represent the SM and the grid-connected inverter are detailed. Then, based on these models, the proposed controller of the VSG is analytically described as the combination of an observer, a linear quadratic regulator (LQR) and an integrator. Finally, as the proposed control is implemented in the control board of an industrial inverter, experimental results are shown to validate the operational behavior of the proposed control architecture for a VSG supplying nonlinear and unbalanced loads, as well as surviving short-circuits and operating in parallel with other power sources [22].

\section{Modeling The VSG}

The models of the SM and the inverter are both expressed in the $d q$-frame and in per unit (p.u.). The SM model implemented in the VSG-based inverter has been extracted from [23] and is based on initial developments presented in [24].

The design proposed for the current controller of the VSG has been developed based on a complete SM in order to be as close as possible of the physical behavior of a diesel generator when facing harsh events. This choice is made to ensure a plug and play solution as well as the possibility to keep the

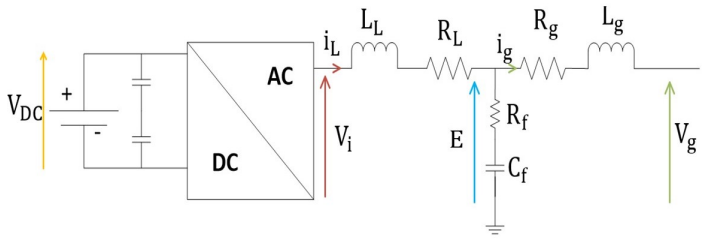

Fig. 1. Single-line inverter's connection to the grid.

same specifications for the industrial developments of VSG as the ones defined in the current norms of diesel generators. In addition, as the proposed current controller has been developed using a state-space model, any other model of SM can be implemented, for instance just the swing equation if ever only virtual inertia is needed.

\section{A. Grid connected inverter}

Fig. 1 depicts the parameters used to model the inverter and its connection to the grid. The inverter's resistor $R_{L}$, the resistor of the capacitive filter $R_{f}$ and the resistor of the grid line $R_{g}$ are not neglected, though small compared to the inverter's reactances $\omega_{\text {base }} . L_{L}, 1 /\left(\omega_{\text {base }} . C_{f}\right)$ and the grid's line reactance $\omega_{\text {base }} . L_{g}$. Hence, the traditionally used LCL output filter is not considered here and instead a RL-RC-RL output inverter filter is preferred for an increased accuracy. The inverter's outputs are defined in the $d q$-frame by (1)-(3).

$$
\begin{aligned}
& \left\{\begin{array}{l}
\frac{d i_{L}^{d}}{d t}=\omega_{\text {base }}\left(\omega_{r} i_{L}^{q}+\frac{V_{i}^{d}-e^{d}}{L_{L}}-\frac{R_{L}}{L_{L}} i_{L}^{d}\right) \\
\frac{d i_{L}^{q}}{d t}=\omega_{\text {base }} \cdot\left(-\omega_{r} i_{L}^{d}+\frac{V_{i}^{q}-e^{q}}{L_{L}}-\frac{R_{L}}{L_{L}} i_{L}^{q}\right)
\end{array}\right. \\
& \left\{\frac{d i_{g}^{d}}{d t}=\omega_{\text {base }} \cdot\left(\omega_{r} i_{g}^{q}+\frac{e^{d}-V_{g}^{d}}{L_{g}}-\frac{R_{L}}{L_{g}} i_{g}^{d}\right)\right. \\
& \frac{d i_{g}^{q}}{d t}=\omega_{\text {base }} \cdot\left(-\omega_{r} i_{g}^{d}+\frac{e^{q}-V_{g}^{q}}{L_{g}}-\frac{R_{L}}{L_{g}} i_{g}^{q}\right) \\
& \left\{\begin{aligned}
\frac{d e^{d}}{d t}= & \omega_{\text {base }}\left(\omega_{r} \cdot e^{q}+\frac{i_{L}^{d}-i_{g}^{d}}{C_{f}}-\frac{R_{f} \cdot R_{L}}{L_{L}} \cdot i_{L}^{d}+\frac{R_{f} \cdot R_{g}}{L_{g}} \cdot i_{g}^{d}\right. \\
& \left.+\frac{R_{f}}{L_{L}} \cdot V_{i}^{d}+\frac{R_{f}}{L_{g}} \cdot V_{g}^{d}-e^{d} \cdot R_{f}\left(\frac{1}{L_{L}}+\frac{1}{L_{g}}\right)\right) \\
\frac{d e^{q}}{d t}= & \omega_{\text {base }}\left(-\omega_{r} \cdot e^{d}+\frac{i_{L}^{q}-i_{g}^{q}}{C_{f}}-\frac{R_{f} \cdot R_{L}}{L_{L}} \cdot i_{L}^{q}+\frac{R_{f} \cdot R_{g}}{L_{g}} \cdot i_{g}^{q}\right. \\
& \left.+\frac{R_{f}}{L_{L}} \cdot V_{i}^{q}+\frac{R_{f}}{L_{g}} \cdot V_{g}^{q}-e^{q} \cdot R_{f}\left(\frac{1}{L_{L}}+\frac{1}{L_{g}}\right)\right)
\end{aligned}\right.
\end{aligned}
$$

\section{B. Complete model in the state-space}

The proposed controller is based on the concatenation of the SM and the inverter's models. To simplify and rewrite it in a state-space framework, we assume that $\omega_{r}=1$. This assumption is relevant since the dynamic frequency variations of the microgrid is limited to less than $10 \%$ of the nominal value. This leads to the following state-space model:

$$
\left\{\begin{array}{l}
\dot{X}_{S Y S}=A_{S Y S} \cdot X_{S Y S}+B_{S Y S} \cdot U_{S Y S}+G_{S Y S} \cdot W_{S Y S} \\
Y_{S Y S}=C_{S Y S} \cdot X_{S Y S}
\end{array}\right.
$$


Where $X_{S Y S}$ represents the state vector, $U_{S Y S}$ the manipulated inputs, $W_{S Y S}$ the exogenous inputs while $Y_{S Y S}$ stands for the regulated outputs. More precisely:

$$
\begin{gathered}
X_{S Y S}=\left[\psi_{d} \psi_{q} \psi_{f d} \psi_{1 d} \psi_{1 q} i_{L}^{d} i_{L}^{q} e^{d} e^{q} i_{g}^{d} i_{g}^{q}\right]^{t} ; \\
U_{S Y S}=\left[\begin{array}{c}
V_{i}^{d} \\
V_{i}^{q}
\end{array}\right] ; W_{S Y S}=\left[\begin{array}{c}
e_{f d} \\
V_{g}^{d} \\
V_{g}^{q}
\end{array}\right] ; Y_{S Y S}=\left[\begin{array}{c}
i_{d} \\
i_{q}
\end{array}\right]
\end{gathered}
$$

The matrices $A_{S Y S}, B_{S Y S}, G_{S Y S}$ and $C_{S Y S}$ are created concatenating the SM and the inverter's models. For an implementable model, a discrete-time version of the above state-space model is used and defined below:

$$
\left\{\begin{array}{l}
X_{S Y S}^{+}=A_{S Y S}^{s} \cdot X_{S Y S}+B_{S Y S}^{s} \cdot U_{S Y S}+G_{S Y S}^{s} \cdot W_{S Y S} \\
Y_{S Y S}=C_{S Y S}^{s} \cdot X_{S Y S}
\end{array}\right.
$$

Where $X_{S Y S}^{+}$represents the next state-space value of the vector $X_{S Y S}$ as well as the matrices $A_{S Y S}^{s}, B_{S Y S}^{s}, C_{S Y S}^{s}$ and $G_{S Y S}^{s}$ are the discrete state-space matrices of the continuous state-space model, defined in (4).

\section{NeW CONTROLLER DESIGN FOR THE VSG}

The objective of the control is to force the system to follow the reference currents defined by the SM model in various configurations of load variations. Active and reactive loads are considered, varying from $0 \%$ to $100 \%$, as well as unbalanced and nonlinear loads and short-circuits. The proposed current controller solution can be divided in two parts: the observer and the controller [25]. The observer reconstructs the output load while the current controller uses the estimated/reconstructed load and the references in order to choose the values of the manipulated variables, helping to supply the necessary output currents to the load should the estimation of the latter be correct.

\section{A. Observer}

The objective of the observer is to reconstruct, based on the available measures, the load variations at the output of the filter, characterized by the grid output voltages $V_{g}^{d}$ and $V_{g}^{q}$ and the grid output currents $i_{g}^{d}$ and $i_{g}^{q}$.

The proposed observer is based on the SM and the inverter models in order to minimize the impact of the monitoring errors that could be introduced on the voltages and currents. The reduction of the number of observer states also reduces the robustness of the entire controller to sensors errors or signal perturbations, justifying our choice (experimentally validated, though not presented in the paper). Hence, as the SM and the average voltage regulation (AVR) are virtual, the fluxes, the SM currents $i_{d}$ and $i_{q}$ and the excitation voltage $e_{f d}$ are available for the observer. In addition, the output inverter currents $i_{L}^{d}$ and $i_{L}^{q}$, the voltages $e^{d}$ and $e^{q}$ are also measured by dedicated sensors. Hence, the outputs vector $Y_{\text {meas }}$, aggregating all measurements, is defined by:

$$
Y_{\text {meas }}=\left[i_{d} i_{q} \psi_{d} \psi_{q} \psi_{f d} \psi_{1 d} \psi_{1 q} i_{L}^{d} i_{L}^{q} e^{d} e^{q} e_{f d}\right]^{t}
$$

And as a linear combination of state and input vectors:

$$
\begin{aligned}
& Y_{\text {meas }}=C_{\text {meas }}^{s} \cdot X_{S Y S}+H_{\text {meas }}^{s} \cdot W_{S Y S} ; \\
& \text { With } C_{\text {meas }}^{s}=\left[\begin{array}{cc}
C_{S Y S}^{s} \\
\mathbb{I}_{9} & \mathbb{O}_{9,2} \\
\mathbb{O}_{1,11}
\end{array}\right] \text { and } H_{\text {meas }}^{s}=\left[\begin{array}{ccc}
\mathbb{O}_{11,3} \\
1 & 0 & 0
\end{array}\right]
\end{aligned}
$$

The exogenous input $W_{S Y S}$ is considered as a constant during multiple periods so we have $W_{S Y S}^{+}=W_{S Y S}$. The model considered for the discrete observer is thus:

$$
\begin{aligned}
& \left\{\begin{array}{l}
\bar{X}_{o b s}^{+}=\bar{A}_{o b s}^{s} \cdot \bar{X}_{o b s}+\bar{B}_{o b s}^{s} \cdot U_{S Y S} \\
Y_{\text {meas }}=\bar{C}_{o b s}^{s} \cdot \bar{X}_{o b s}
\end{array}\right. \\
& \text { With: } \bar{X}_{o b s}=\left[\begin{array}{c}
X_{S Y S} \\
W_{S Y S}
\end{array}\right] ; \bar{A}_{o b s}^{s}=\left[\begin{array}{cc}
A_{S Y S}^{s} & G_{S Y S}^{s} \\
\mathbb{O}_{3,11} & \mathbb{I}_{3}
\end{array}\right] ; \\
& \bar{B}_{o b s}^{s}=\left[\begin{array}{c}
B_{S Y S}^{s} \\
\mathbb{O}_{3,2}
\end{array}\right] \text { and } \bar{C}_{o b s}^{s}=\left[\begin{array}{ll}
C_{S Y S}^{s} & H_{S Y S}^{s}
\end{array}\right]
\end{aligned}
$$

Fortunately, the new extended model is observable. Therefore, it is possible to compute a matrix gain $L_{o b s}$ of a linear state observer, which is written as follows, with $L_{\text {obs }}$ a combination of $L_{o b s}^{X}$ and $L_{o b s}^{W}$ :

$$
\begin{aligned}
& \left\{\begin{array}{l}
\hat{\bar{X}}_{o b s}^{+}=\left(\bar{A}_{o b s}^{s}-L_{o b s} \cdot \bar{C}_{o b s}^{s}\right) \cdot \hat{\bar{X}}_{o b s} \\
\quad+\bar{B}_{o b s}^{s} \cdot U_{S Y S}+L_{o b s} \cdot Y_{\text {meas }} \\
\hat{Y}_{\text {meas }}=\bar{C}_{o b s}^{s} \cdot \hat{\bar{X}}_{o b s}
\end{array}\right. \\
& \text { With: } L_{o b s}=\left[\begin{array}{cc}
L_{X}^{X} & L_{W}^{X} \\
L_{X}^{W} & L_{W}^{W}
\end{array}\right]
\end{aligned}
$$

With the integration of a state observer in the proposed controller, the VSG-based inverter is capable to determine the characteristics of any unpredictable load consumption and adjust automatically to changes in the microgrid. The entire proposed controller is mathematically detailed, which does not constitute a proof per say of the importance of the observer, but is what was possible within pages limitations.

\section{B. Output feedback controller}

Let us first introduce the integrator that enables to recover long range errors in steady state possibly due to modeling.

1) Integrator: The LQR controller's output is the difference between the inverter's currents, $i_{L}^{d}$ and $i_{L}^{q}$, and the references, the SM's currents $i_{d}$ and $i_{q}$. Hence, the output $\varepsilon$ is defined as:

$$
\begin{aligned}
\varepsilon=\left[\begin{array}{l}
i_{d}-i_{L}^{d} \\
i_{q}-i_{L}^{q}
\end{array}\right]=C_{\varepsilon}^{s} \cdot \bar{X}_{S Y S} & \\
& \text { With } C_{\varepsilon}^{s}=C_{S Y S}^{s}-\left[\begin{array}{lll}
\mathbb{O}_{2,5} & \mathbb{I}_{2} & \mathbb{O}_{2,4}
\end{array}\right]
\end{aligned}
$$

In order to ensure a proper rejection of the disturbances, an integration state is added to the current controller with the following dynamics:

$$
\varepsilon_{\text {Int }}^{+}=\left[\begin{array}{l}
\varepsilon_{\text {Int }}^{d} \\
\varepsilon_{\text {Int }}^{q}
\end{array}\right]^{+}=\left[\begin{array}{l}
\varepsilon_{\text {Int }}^{d} \\
\varepsilon_{\text {Int }}^{q}
\end{array}\right]+\varepsilon
$$


Considering the extended state $\bar{X}_{S Y S}=\left[\begin{array}{c}X_{S Y S} \\ \varepsilon_{\text {Int }}\end{array}\right]$, obtained by including $\varepsilon_{I n t}$ and the matrix $C_{\varepsilon}^{s}$ from (11) and (12), the new extended model can be written from (6):

$$
\begin{aligned}
& \left\{\begin{array}{l}
\bar{X}_{S Y S}^{+}=\bar{A}_{S Y S}^{s} \cdot \bar{X}_{S Y S}+\bar{B}_{S Y S}^{s} \cdot U_{S Y S}+\bar{G}_{S Y S}^{s} \cdot W_{S Y S} \\
\varepsilon=C_{\varepsilon}^{s} \cdot \bar{X}_{S Y S}
\end{array}\right. \\
& \text { With } \bar{A}_{S Y S}^{s}=\left[\begin{array}{cc}
A_{S Y S}^{s} & \mathbb{O}_{11,2} \\
C_{\varepsilon}^{s} & \mathbb{I}_{2}
\end{array}\right] ; \\
& \bar{B}_{S Y S}^{s}=\left[\begin{array}{c}
B_{S Y S}^{s} \\
\mathbb{O}_{2,2}
\end{array}\right] \text { and } \bar{G}_{S Y S}^{s}=\left[\begin{array}{c}
G_{S Y S}^{s} \\
\mathbb{O}_{2,3}
\end{array}\right]
\end{aligned}
$$

2) Design of the controller system: The equations in steadystates of the system, (12) and (13), gives:

$$
\begin{aligned}
{\left[\begin{array}{cc}
\bar{G}_{S Y S}^{s} & \mathbb{O}_{13,2} \\
\mathbb{O}_{2,3} & \mathbb{I}_{2}
\end{array}\right] \cdot\left[\begin{array}{c}
W_{S Y S} \\
\varepsilon^{*}
\end{array}\right] } \\
=\underbrace{\left[\begin{array}{cc}
\mathbb{I}_{13}-\bar{A}_{S Y S}^{s} & \bar{B}_{S Y S}^{s} \\
C_{\varepsilon}^{s} & \mathbb{O}_{2,2}
\end{array}\right]}_{\mathbb{F}} \cdot\left[\begin{array}{c}
X_{S Y S} \\
U_{S Y S}
\end{array}\right]
\end{aligned}
$$

Since the matrix $\mathbb{F}$ can be inverted, the steady-states values $\bar{X}_{S Y S}^{*}$ and $U_{S Y S}^{*}$, that depend on $W_{S Y S}$ and $\varepsilon^{*}$, can be obtained with:

$$
\left[\begin{array}{l}
\bar{X}_{S Y S}^{*} \\
U_{S Y S}^{*}
\end{array}\right]=\underbrace{\mathbb{F}^{-1} \cdot\left[\begin{array}{cc}
\bar{G}_{S Y S}^{s} & \mathbb{O}_{13,2} \\
\mathbb{O}_{2,3} & \mathbb{I}_{2}
\end{array}\right]}_{\left[\begin{array}{ll}
K_{W}^{X} & K_{\epsilon}^{X} \\
K_{W}^{U} & K_{\epsilon}^{U}
\end{array}\right]} \cdot\left[\begin{array}{c}
W_{S Y S} \\
\varepsilon^{*}
\end{array}\right]
$$

Where we define a matrix coefficients used in the following equations. Eq. (15) enables to write the dynamics of the deviation variables as follows:

$$
\left\{\begin{array}{c}
\left(\bar{X}_{S Y S}-\bar{X}_{S Y S}^{*}\right)^{+}=\bar{A}_{S Y S}^{s} \cdot\left(\bar{X}_{S Y S}-\bar{X}_{S Y S}^{*}\right) \\
+\bar{B}_{S Y S}^{s} \cdot\left(U_{S Y S}-U_{S Y S}^{*}\right) \\
\varepsilon-\varepsilon^{*}=C_{\varepsilon}^{s} \cdot\left(\bar{X}_{S Y S}-\bar{X}_{S Y S}^{*}\right)
\end{array}\right.
$$

The expression of (16) can be simplified as:

$$
\begin{gathered}
\left\{\begin{array}{l}
\bar{\varepsilon}_{X}{ }^{+}=\bar{A}_{S Y S}^{s} \cdot \bar{\varepsilon}_{X}+\bar{B}_{S Y S}^{s} \cdot \varepsilon_{U} \\
\varepsilon_{Y}=C_{\varepsilon}^{s} \cdot \varepsilon_{X}
\end{array}\right. \\
\text { With: } \bar{\varepsilon}_{X}=\bar{X}_{S Y S}-\bar{X}_{S Y S}^{*} ; \\
\varepsilon_{U}=\left(U_{S Y S}-U_{S Y S}^{*}\right) \text { and } \varepsilon_{Y}=\varepsilon-\varepsilon^{*}
\end{gathered}
$$

3) Current controller: To avoid the saturation of the inverter's duty ratio, the controller is not directly applied on the command $U_{S Y S}$ but on its variation $\Delta U_{S Y S}$. The application of the controller on the variation of the command $\triangle U_{S Y S}$ permits to smooth the variation of the command $U_{S Y S}$ and avoids any discontinuity. Thanks to that, the risk of harsh variations of the command $U_{S Y S}$ during transitory events is minimized, thus reducing the risk of saturation. add reference?

So, the state space $U_{S Y S}$ is added to the model: $U_{S Y S}{ }^{+}=$ $U_{S Y S}+\Delta U$. The variable $\Delta U$ is the input of the system and as $\varepsilon_{U}=U_{S Y S}-U_{S Y S}^{*}$, the new state is $\varepsilon_{U}^{+}=\varepsilon_{U}+\Delta U$. Hence, from (17), the updated version of the model, including $\varepsilon_{U}$ as a state is expressed in (18).

$$
\left\{\begin{array}{l}
{\left[\begin{array}{l}
\bar{\varepsilon}_{X} \\
\varepsilon_{U}
\end{array}\right]^{+}=\left[\begin{array}{cc}
\bar{A}_{S Y S}^{s} & \bar{B}_{S Y S}^{s} \\
\mathbb{O}_{2,13} & \mathbb{I}_{2}
\end{array}\right] \cdot\left[\begin{array}{c}
\bar{\varepsilon}_{X} \\
\varepsilon_{U}
\end{array}\right]+\left[\begin{array}{c}
\mathbb{O}_{13,2} \\
\mathbb{I}_{2}
\end{array}\right] \cdot \Delta U} \\
\varepsilon_{Y}=\left[\begin{array}{ll}
C_{\varepsilon}^{s} & \mathbb{O}_{2,2}
\end{array}\right] \cdot\left[\begin{array}{c}
\bar{\varepsilon}_{X} \\
\varepsilon_{U}
\end{array}\right]
\end{array}\right.
$$

Based on the dynamical model (18), it is possible to control the inverter's state using a standard LQR design that takes the following form:

$$
\Delta U=-K^{s} \cdot\left[\begin{array}{l}
\bar{\varepsilon}_{X} \\
\varepsilon_{U}
\end{array}\right]
$$

From (18) and (19), the controlled system is defined, around a steady-state value in (20).

$$
\begin{aligned}
& \left\{\begin{array}{l}
{\left[\begin{array}{c}
\bar{\varepsilon}_{X} \\
\varepsilon_{U}
\end{array}\right]^{+}=\underline{A}^{s} \cdot\left[\begin{array}{c}
\bar{\varepsilon}_{X} \\
\varepsilon_{U}
\end{array}\right]} \\
{\left[\begin{array}{c}
\varepsilon_{Y} \\
\Delta U
\end{array}\right]=\left[\begin{array}{c}
\underline{C}^{s} \\
-K^{s}
\end{array}\right] \times\left[\begin{array}{l}
\bar{\varepsilon}_{X} \\
\varepsilon_{U}
\end{array}\right]}
\end{array}\right. \\
& \text { With }\left\{\begin{array}{l}
\underline{A}^{s}=\left[\begin{array}{cc}
\bar{A}_{S Y S}^{s} & \bar{B}_{S Y S}^{s} \\
\mathbb{O}_{2,13} & \mathbb{I}_{2}
\end{array}\right]-\left[\begin{array}{c}
\mathbb{O}_{13,2} \\
\mathbb{I}_{2}
\end{array}\right] \cdot K^{s} \\
\underline{C}^{s}=\left[\begin{array}{ll}
C_{\varepsilon}^{s} & \mathbb{O}_{2,2}
\end{array}\right]
\end{array}\right.
\end{aligned}
$$

Hence, considering the definition of the states variables $\bar{\varepsilon}_{X}$, $\varepsilon_{U}, \varepsilon_{Y}$ and the characteristics of the reference values $\bar{X}_{S Y S}^{*}$ and $U_{S Y S}^{*}$, the controlled system can also be expressed in (21).

$$
\left\{\begin{aligned}
{\left[\begin{array}{r}
\bar{X}_{S Y S} \\
U_{S Y S}
\end{array}\right]^{+}=\underline{A}^{s} \cdot\left[\begin{array}{l}
\bar{X}_{S Y S} \\
U_{S Y S}
\end{array}\right] } \\
-\left(\underline{A}^{s}-\mathbb{I}_{15}\right) \cdot\left[\begin{array}{ll}
K_{W}^{X} & K_{\varepsilon}^{X} \\
K_{W}^{U} & K_{\varepsilon}^{U}
\end{array}\right] \cdot\left[\begin{array}{c}
W_{S Y S} \\
\varepsilon^{*}
\end{array}\right] \\
{\left[\begin{array}{c}
\varepsilon \\
\Delta U
\end{array}\right]=\left[\begin{array}{c}
\underline{C}^{s} \\
-K^{s}
\end{array}\right] \cdot\left[\begin{array}{c}
\bar{X}_{S Y S} \\
U_{S Y S}
\end{array}\right]-\left(\left[\begin{array}{c}
\underline{C}^{s} \\
-K^{s}
\end{array}\right] \cdot\right.} \\
{\left.\left[\begin{array}{ll}
K_{W}^{X} & K_{\varepsilon}^{X} \\
K_{W}^{U} & K_{\varepsilon}^{U}
\end{array}\right]-\left[\begin{array}{ll}
\mathbb{O}_{2,3} & \mathbb{I}_{2} \\
\mathbb{O}_{2,3} & \mathbb{O}_{2,2}
\end{array}\right]\right) \cdot\left[\begin{array}{c}
W_{S Y S} \\
\varepsilon^{*}
\end{array}\right] }
\end{aligned}\right.
$$

\section{Complete model}

The extended system including the observer, in which the controlled input is the increment $\Delta U$, is determined by the equations (10) and (21). Its schematic representation is proposed in Fig. 2 and the final expression in (22). The predicted states are used in the complete controller in order to minimize the impact of the monitoring errors that could be introduced by the voltage and current captors, thus increasing the robustness of the proposed controller. 


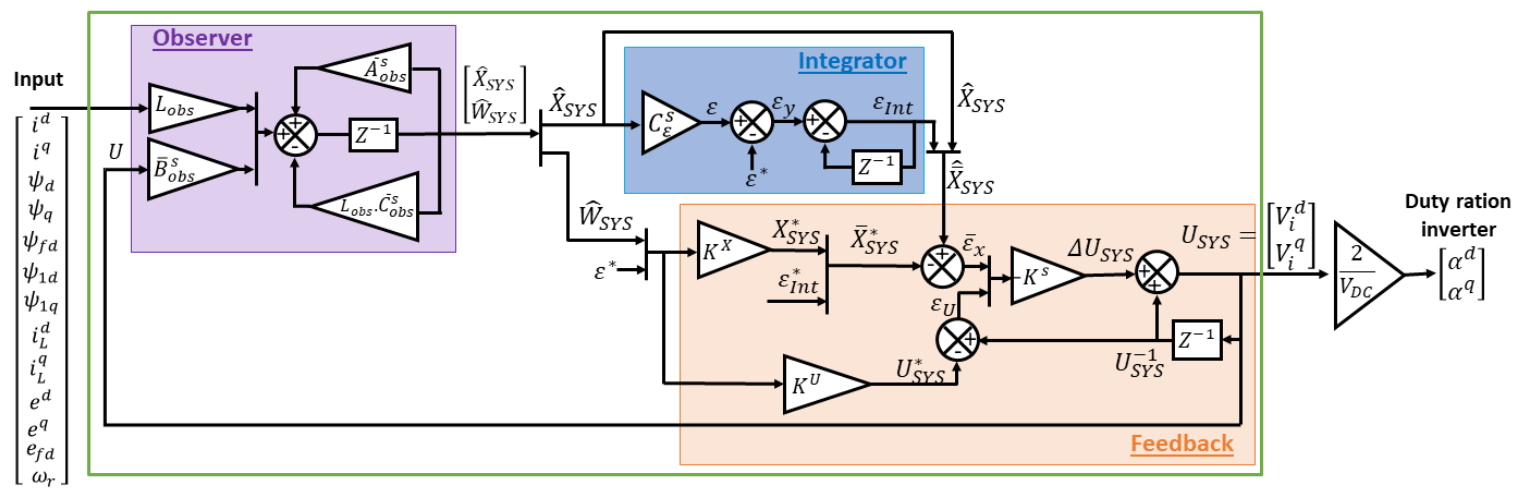

Fig. 2. Controller proposed synoptic, with $K^{X}=\left[\begin{array}{cc}K_{W}^{X} & K_{\varepsilon}^{X}\end{array}\right]$ and $K^{U}=\left[\begin{array}{ll}K_{W}^{U} & K_{\varepsilon}^{U}\end{array}\right]$ (from (15))

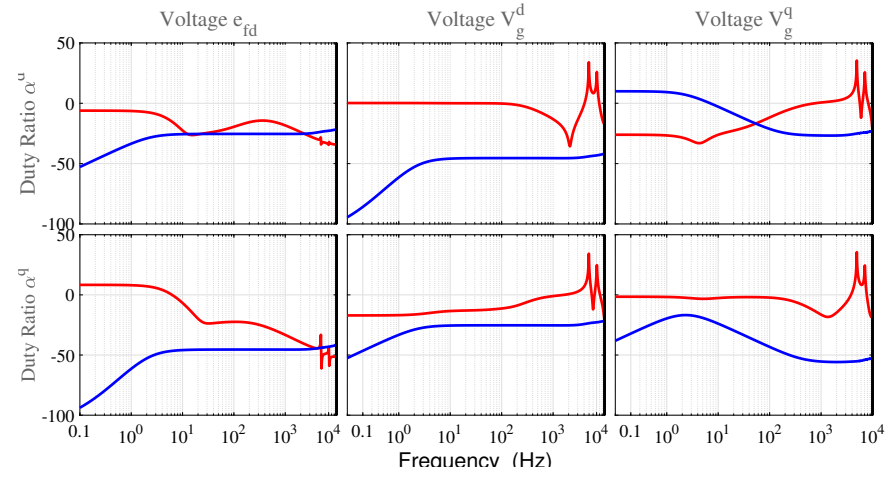

Fig. 3. Bode magnitude diagram of the proposed controller (in blue) compared to a "PI+filters" implemented from [24] (in red).

$$
\begin{aligned}
& \left\{\begin{array}{l}
\widehat{X}_{\Delta}^{+}=A_{\Delta} \cdot \widehat{X}_{\Delta}+B_{\Delta} \cdot\left[\begin{array}{c}
Y_{\text {meas }} \\
\varepsilon^{*}
\end{array}\right] \\
\widehat{Y}_{\Delta}=C_{\Delta} \cdot \widehat{X}_{\Delta}+D_{\Delta} \cdot\left[\begin{array}{c}
Y_{\text {meas }} \\
\varepsilon^{*}
\end{array}\right]
\end{array}\right. \\
& \text { In which: } X_{\Delta}=\left[\begin{array}{c}
\bar{X}_{S Y S} \\
U_{S Y S} \\
W_{S Y S}
\end{array}\right] ; Y_{\Delta}=\left[\begin{array}{c}
Y_{\text {meas }} \\
\varepsilon \\
\Delta U
\end{array}\right] \\
& L_{o b s \Delta}=\left[\begin{array}{ccc}
L^{X} & \mathbb{O}_{11,4} & L_{W}^{X} \\
\mathbb{O}_{4,11} & \mathbb{O}_{4,4} & \mathbb{O}_{4,3}^{W} \\
L_{X}^{W} & \mathbb{O}_{3,4} & L_{W}^{W}
\end{array}\right] ; \\
& A_{\Delta}=\left[\begin{array}{cc}
\underline{A}^{s} & -\left(\underline{A}^{s}-\mathbb{I}_{15}\right) \cdot\left[\begin{array}{l}
K_{W}^{X} \\
K_{W}^{U}
\end{array}\right] \\
\mathbb{O}_{3,15} & \mathbb{I}_{3}
\end{array}\right] \\
& \text { - } L_{\text {obs } \Delta} \cdot\left[\begin{array}{lll}
C_{\text {meas }} & \mathbb{O}_{12,4} & H_{\text {meas }}
\end{array}\right] \text {; }
\end{aligned}
$$

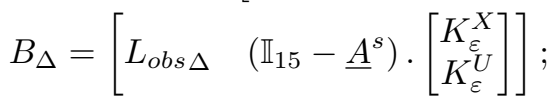

$$
\begin{aligned}
& C_{\Delta}=\left[\begin{array}{cc}
{\left[\begin{array}{cc}
C_{\text {meas }}^{s} & \mathbb{O}_{4,12}
\end{array}\right]} & H_{\text {meas }}^{s} \\
\underline{C}^{s} & -\underline{C}^{s} \cdot\left[\begin{array}{l}
K_{W}^{X} \\
K_{W}^{U}
\end{array}\right] \\
-K^{s} & K^{s} \cdot\left[\begin{array}{l}
K_{W}^{X} \\
K_{W}^{U}
\end{array}\right]
\end{array}\right] ; \\
& D_{\Delta}=\left[\begin{array}{cc}
\mathbb{O}_{12,12} & \mathbb{O}_{12,2} \\
\mathbb{O}_{2,12} & \mathbb{I}_{2}-\underline{C}^{s} \cdot\left[\begin{array}{l}
K_{\varepsilon}^{X} \\
K_{\varepsilon}^{U}
\end{array}\right] \\
\mathbb{O}_{2,12} & K^{s} \cdot\left[\begin{array}{l}
K_{\varepsilon}^{X} \\
K_{\varepsilon}^{U}
\end{array}\right]
\end{array}\right]
\end{aligned}
$$

\section{Choice of the controller}

The proposed controller, a LQR integrating a state observer and an integral functionality, has been chosen to extend the operational range of the VSG, to better represent the ideal behavior of a SM. Thanks to the addition of the integrator in controller, errors that can be introduced by the observer (delay or monitoring errors for instance) are minimized by the integral part of the output feedback. This phenomenon is illustrated in Fig. 3, where the reader can see that the low frequencies are rejected by the controller.

In addition, similarly to diesel generators, VSGs will be submitted to sudden and almost unpredictable load variations at the microgrid level due to a low power reserve. Hence, the proposed controller is designed as a solution to unpredictable characteristic of loads consumption and characteristics (including its variation in time).

Fig. 3 represents the Bode magnitude diagram of the proposed controller compared to a controller based on a "PI+filters" implementation used in a similar context [24]. The voltages inputs are presented in function of the duty ratio outputs.

It can be noted that the "PI+filters" implementation posses a resonance peak at high frequency, meaning that the system could be unstable during high frequency phenomena [3], especially during short-circuits. This instability has been observed experimentally when submitting our VSG-based solution with highly inductive loads with the previously implemented controller. As no such high frequency resonance is visible with the proposed controller, thanks to the state observer and the integrator, the proposed controller is stable during the similar experimental load impacts. In addition, the proposed controller rejects low frequencies as well, which minimizes the impact of DC components on the operational stability of the VSG solution.

\section{EXPERIMENTAL VALIDATION}

The described model has been experimentally validated on a large variety of loads (including variable and nonlinear ones as well as short-circuits). In order to represent the unpredictability of the loads variations, we decided to test step variations of $100 \%$. Indeed, this "worst case scenario" allows us, by 


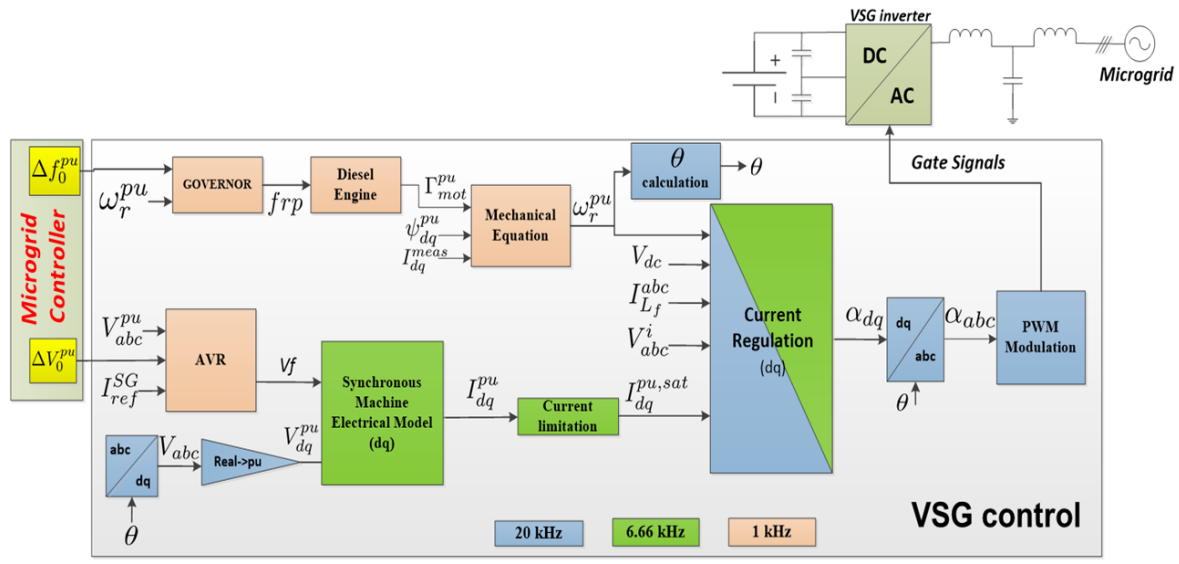

Fig. 4. Complete VSG control diagram.

definition, to validate the proper operation of the proposed controller for all the others uses cases.

\section{A. Experimental facility}

The proposed controller for the VSG was tested within the Schneider Electric R\&D center in Grenoble (France), detailed in [24]. This is a dedicated microgrid laboratory including several power sources that can be put in parallel on a $100 \mathrm{kVA}$ distribution grid. There are two categories of power sources: diesel generators $(15 \mathrm{kVA}, 45 \mathrm{kVA}$ and $50 \mathrm{kVA}$ ) and four inverter-based power sources (Schneider Electric Solar inverters of $25 \mathrm{kVA}$ ), two of which are in a grid-tie configuration with solar emulators (controllable DC power source), and are operated using the VSG-based control. Several loads are connected to the distribution grid: RLC loads, industrial load (induction motors), a compressor and a motor controlled by a $15 \mathrm{kVA}$ drive. Finally, there is a shortcircuit cabinet with various combinations (single-phase, phasephase, tri-phase) with a short or long time duration.

\section{B. Implementation of the proposed controller}

The proposed current controller, a linear quadratic regulator incorporating an integrator and a state-observer, was integrated to the complete VSG control as presented in Fig. 4. The implementation in the digital signal processor (DSP) is straightforward as the proposed controller is already discrete. The model is implemented thanks to Matlab/Simulink ${ }^{\mathrm{TM}}$ using the "Embedded Coder" toolbox of Mathworks" ${ }^{\mathrm{TM}}$, and the "Code Composer Studio" toolkit to the inverter's DSP (a TMS320F28335, Texas Instrument ${ }^{\mathrm{TM}}$ ). Note that all parts of the VSG control visible in Fig. 4 are not detailed in this paper due to page limitation, but are presented in [24].

\section{Experimental Results}

During the experiments, multiple scenarios were conducted to validate the proposed VSG controller and its acceptable dynamic behavior: a "traditional" load impact, nonlinear and unbalanced loads as well as short-circuits were investigated in addition to operation in parallel with other power sources.
Note that nonlinear loads and short-circuits are not commonly tested for VSG although they should be mandatory before considering any further industrial development and standardization.

Note that the steady states is not reached very fast in Fig. 5 to Fig.10 because the embedded synchronous machine model and the time response characteristics implemented a reference were designed so for easier observations. It is not due to the performances of the current controller and could be adapted.

1) Load impact: Fig. 5 shows the dynamic behavior of the VSG supplying power to a $20 \mathrm{~kW}$ load (impact of $100 \%$ of the load). Voltages and currents are similar to an actual SM with a sub-transient and transient phenomenon before stabilizing to a steady-state. Because of the AVR and the droop control, the VSG stabilizes to a new voltage value while supplying the load.

2) Nonlinear and unbalanced load impacts: To consider an industrial development, the VSG should be capable of supplying any profile of load, including unbalanced or nonlinear ones, especially motors or drives. Note that these different tests are generally not considered for the system validation in the literature and represent a plus for the proposed controller validation in the present paper.

Indeed, industrial grid-connected or off-grid facilities represent an important part of the current microgrids. To this respect, Fig. 7 and Fig. 6 show that, with the proposed controller, the VSG remains stable and is able to supply significantly unbalanced or nonlinear loads such as a $3 \mathrm{~kW}$ single-phase motor or a $15 \mathrm{kVA}$ drive.

3) Short-circuit: With the increase in connection of inverter-based generators (IBG) in microgrids, another problem could also appear: the way protections are handling shortcircuits. Indeed, the inverter's output current is limited between 1.2 to 2 times the nominal value and cannot compete with the short-circuit current of a real SM (more than 10 time the nominal current). With IBG, the currents will then be saturated. Hence, given that the short-circuit prediction is difficult, the VSG must be stable during a standard version of this fault to help protections operating properly. To this respect, Fig. 8 and Fig. 9 show the behavior of the VSG with the proposed controller facing two short-circuits: a tri-phase 


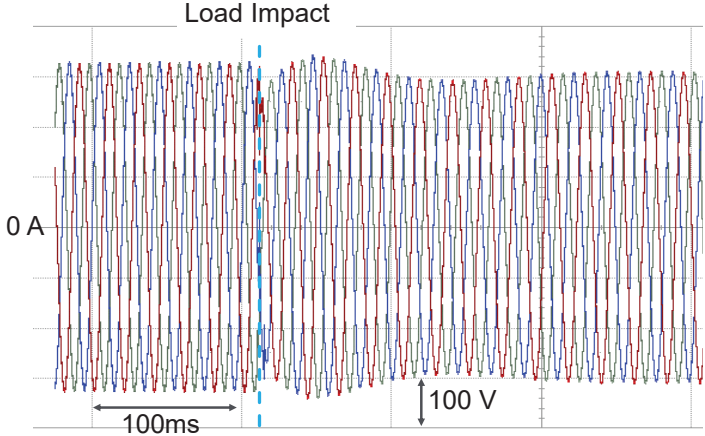

(a) Voltage.

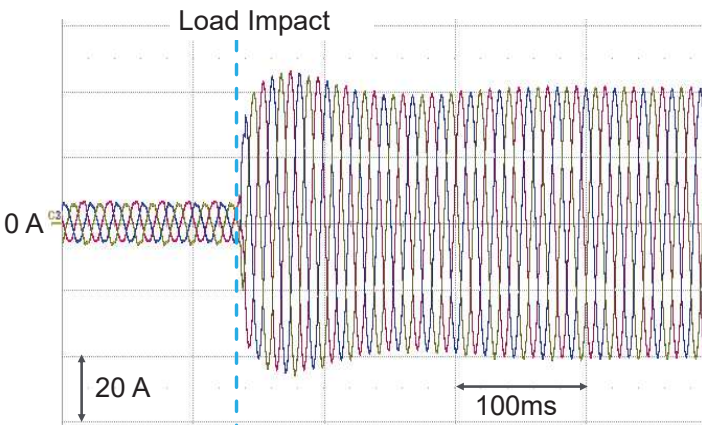

(b) Current.

Fig. 5. $20 \mathrm{~kW}$ load impact on the VSG.

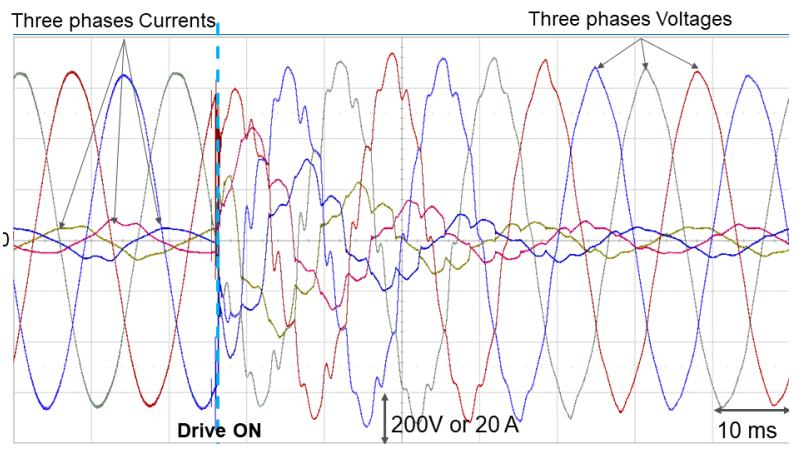

Fig. 6. VSG supplying a $15 \mathrm{kVA}$ drive connected to a DC machine.

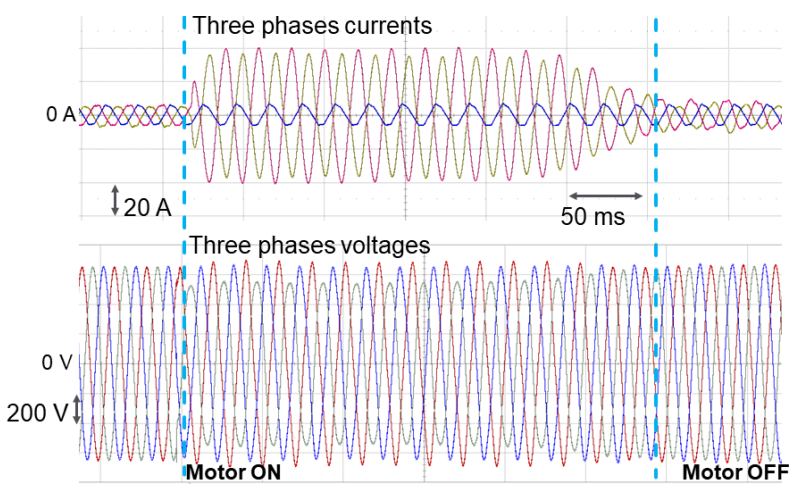

Fig. 7. VSG supplying a $3 \mathrm{~kW}$ single-phase motor.

one and a phase-phases one.

Fig. 8 is a focus on the beginning of the tri-phase shortcircuit. The VSG's voltage is null and current oscillations can be seen due to the impedance of the output LCL filter

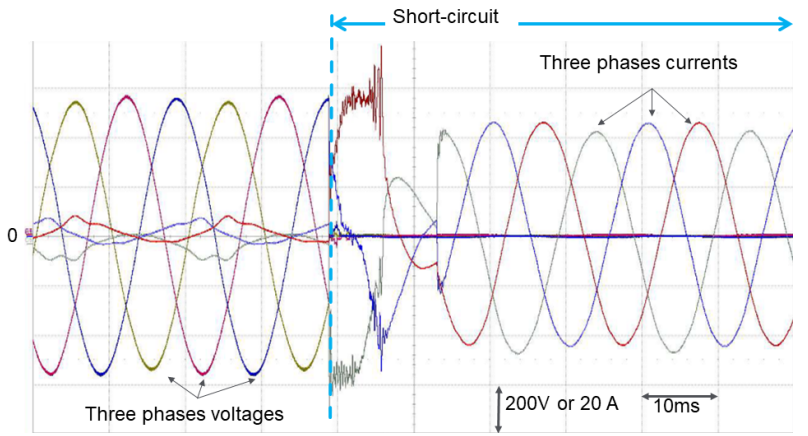

Fig. 8. VSG behavior during a tri-phase short-circuit.

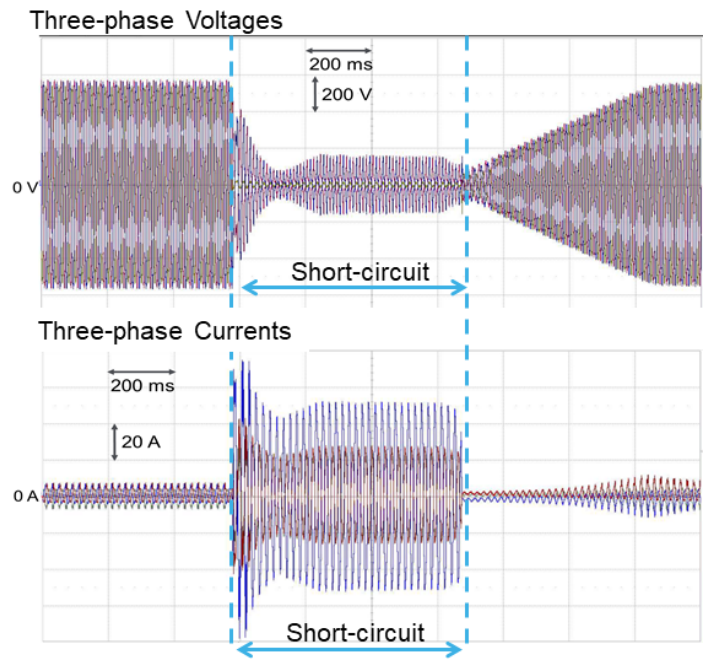

Fig. 9. VSG behavior during a phase-phase short-circuit.

and the transformer. Similarly to the phase-phase short-circuit, the short-circuit currents stabilize after $15 \mathrm{~ms}$, reaching the maximal current of the inverter.

Fig. 9 shows the behavior of the VSG during the phasephases short-circuit, with the voltage increasing back to its nominal value after the fault. The maximal current is reached after $200 \mathrm{~ms}$. As the short-circuit is between two phases, currents are equal in those phases. The current of the third phase is the opposite of the sum of these two currents.

Note that the VSG-based controller is able to sustain those short-circuits for an infinite duration, theoretically speaking. The only constraint is that the model should remain stable, which will always be the case, except possibly for a too short duration (in our case, around a few milliseconds) where the saturation of the inverter could destabilize its operation.

4) Parallel operation with a generator set: The VSG is developed as a plug-and-play solution. As such, it must operate in parallel with other power sources. The microgrid instabilities can be exacerbated by the resonance among generators and VSG [26]. Hence, we should validate the VSG parallel operation with other power sources. In Fig. 10, a 17.5 kVA highly inductive load is connected to an already supplied $15 \mathrm{~kW}$ load by a $25 \mathrm{kVA}$ VSG and $45 \mathrm{kVA}$ generator set.

When the load is connected to the microgrid, both power sources produce an peak current due to the inductive nature 


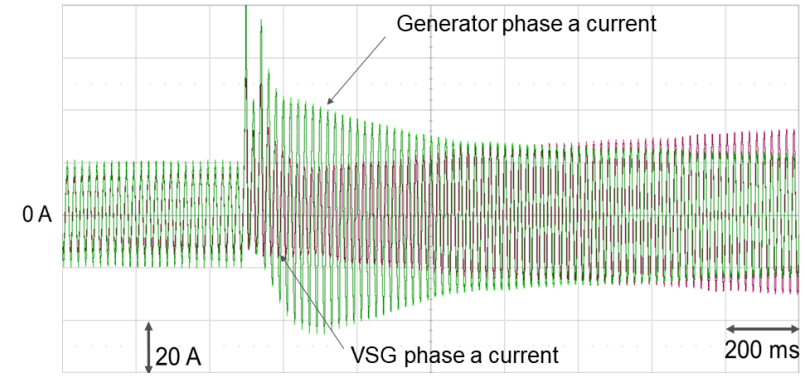

Fig. 10. Current response (phase $a$ ) of the VSG and generator set while supplying a $15 \mathrm{~kW}$ load and facing a $17.5 \mathrm{kVA}$ inductive load impact.

of the load impact. The VSG rapidly absorbs the DC current (in less than $200 \mathrm{~ms}$ ). This is not the case of the traditional generator, which takes more than $400 \mathrm{~ms}$ with the used controller. Once the DC current is dissipated, as the generator set has a limited reactive power capacity, the VSG supplies most of the reactive power needed by the inductive load. Both power sources supply the loads and the voltage remains stable at a new steady-state value that depends on the droop control, without oscillations.

\section{CONCLUSiON}

In this paper, a control architecture based on the state-space model of a synchronous machine (SM) and the grid-connected inverter, combined with an observer, is proposed to enhance the stability of an inverter-based generator operated as a virtual synchronous generator (VSG) in a microgrid. VSGs are submitted to sudden and almost unpredictable load variations at the microgrid level. Hence, the proposed controller has been designed as a solution to face the unpredictability of loads consumption thus decreasing risks of instability.

First, the state-space model is expressed for both the SM and the grid-connected inverter. Second, the controller for the VSG is proposed, including an observer based on the gridconnected inverter. Moreover, a LQR controller, designed with an integrator based on the entire SM combined with the gridconnected inverter is proposed, enforcing the control of the VSG-based inverter. This allows the VSG solution to survive harsh events like short-circuits and to still present a behavior as close as possible to a diesel generator (i.e.not only providing virtual inertia). From the industrial point of view, the solution is then plug and play, necessitating very little to satisfy diesel generators current standards, thus enforcing its capacity to be more easily accepted by customers used to more traditional generators.

Finally, the paper concludes on experimental results validating the relevance and the robustness of the control of the VSG, facing significant impacts of R, L, nonlinear and unbalanced loads (which are not traditionally found in studies on VSG) as well as short-circuits (tri-phase and phase-phase) and parallel operation (with a generator set).

Thanks to the proposed controller, the VSG is stable and is capable of supplying highly nonlinear or unbalanced loads such as drives or single-phase motors, two types of shortcircuits, and operate in parallel with a generator set supplying a compressor.
Undergoing works consider taking advantages of the virtual part of the VSG in order to increase its performance by modifying artificially the parameters of the SM model to improve the VSG inverter performances and also to avoid the deterioration of the inverter during harsh events.

\section{REFERENCES}

[1] A. Hoke, R. Butler, J. Hambrick, and B. Kroposki, "Maximum Photovoltaic Penetration Levels on Typical Distribution Feeders," IEEE Transactions on Sustainable Energy, no. July, 2012.

[2] J. Driesen and K. Visscher, "Virtual synchronous generators," in IEEE Power and Energy Society General Meeting - Conversion and Delivery of Electrical Energy in the 21st Century, no. August 2008. Pittsburgh: IEEE, Jul. 2008, pp. 1-3.

[3] H. Alatrash, A. Mensah, E. Mark, G. Haddad, and J. Enslin, "Generator Emulation Controls for Photovoltaic Inverters," IEEE Transactions on Smart Grid, vol. 3, no. 2, pp. 996-1011, Jun. 2012.

[4] Y. Chen, R. Hesse, D. Turschner, and H.-P. Beck, "Comparison of methods for implementing virtual synchronous machine on inverters," in International Conference on Renewable Energies and Power Qualities, vol. 1, no. 10, 2012, pp. 734-739.

[5] S. D'Arco, J. A. Suul, and O. B. Fosso, "A Virtual Synchronous Machine implementation for distributed control of power converters in SmartGrids," Electric Power Systems Research, vol. 122, pp. 180-197, May 2015.

[6] Y. Hirase, K. Sugimoto, K. Sakimoto, and T. Ise, "Analysis of resonance in microgrids and effects of system frequency stabilization using a virtual synchronous generator," IEEE Journal of Emerging and Selected Topics in Power Electronics, vol. 4, no. 4, pp. 1287-1298, 2016.

[7] O. Mo, S. D’Arco, and J. A. Suul, "Evaluation of Virtual Synchronous Machines with Dynamic or Quasi-stationary Machine Models," IEEE Transactions on Industrial Electronics, vol. 64, no. 7, pp. 1-1, 2016.

[8] H. Bevrani, T. Ise, and Y. Miura, "Virtual synchronous generators: a survey and new perspectives," International Journal of Electrical Power \& Energy Systems, vol. 54, pp. 244-254, Jan. 2014.

[9] M. Torres and L. A. C. Lopes, "A virtual synchronous machine to support dynamic frequency control in a mini-grid that operates in frequency droop mode," Energy and Power Engineering, vol. 5, no. 3, pp. 259-265, 2013.

[10] J. Liu, Y. Miura, and T. Ise, "Comparison of Dynamic Characteristics Between Virtual Synchronous Generator and Droop Control in InverterBased Distributed Generators," IEEE Transactions on Power Electronics, vol. 31, no. 5, pp. 3600-3611, May 2016.

[11] M. Li, Y. Wang, Y. Liu, N. Xu, S. Shu, and W. Lei, "Enhanced power decoupling strategy for virtual synchronous generator," IEEE Access, vol. 8, pp. $73601-73613,2020$.

[12] L.-Y. Lu and C.-C. Chu, "Consensus-Based Secondary Frequency and Voltage Droop Control of Virtual Synchronous Generators for Isolated AC Micro-Grids," IEEE Journal on Emerging and Selected Topics in Circuits and Systems, vol. 5, no. 3, pp. 443-455, Sep. 2015.

[13] Q.-C. Zhong, G. C. Konstantopoulos, B. Ren, and M. Krstic, "Improved Synchronverters with Bounded Frequency and Voltage for Smart Grid Integration," IEEE Transactions on Smart Grid, vol. 3053, no. c, pp. $1-1,2016$.

[14] M. A. Torres L., L. A. C. Lopes, L. A. Morán T., and J. R. Espinoza C., "Self-tuning virtual synchronous machine: a control strategy for energy storage systems to support dynamic frequency control," IEEE Transactions on Energy Conversion, vol. 29, no. 4, pp. 833-840, Dec. 2014.

[15] I. Serban and C. P. Ion, "Microgrid control based on a grid-forming inverter operating as virtual synchronous generator with enhanced dynamic response capability," International Journal of Electrical Power \& Energy Systems, vol. 89, pp. 94-105, 2017.

[16] S. D'Arco and J. A. Suul, "Virtual synchronous machines - Classification of implementations and analysis of equivalence to droop controllers for microgrids," in IEEE PowerTech, Grenoble, France, 2013, pp. 1-6.

[17] C. F. Dos Santos, F. B. Grigoletto, and M. Stefanello, "Power quality improvement in a grid connected voltage source inverter using the concept of virtual synchronous machine," in IEEE Brazilian Power Electronics Conference and Southern Power Electronics Conference (COBEP/SPEC). IEEE, Nov. 2015, pp. 1-5.

[18] M. Chen and X. Xiao, "Hierarchical frequency control strategy of hybrid droop/VSG-based islanded microgrids," Electric Power Systems Research, vol. 155, pp. 131-143, 2018. 
[19] C. Andalib-Bin-Karim, X. Liang, and H. Zhang, "Fuzzy-SecondaryController-Based Virtual Synchronous Generator Control Scheme for Interfacing Inverters of Renewable Distributed Generation in Microgrids," IEEE Transactions on Industry Applications, vol. 54, no. 2, pp. 10471061, Mar. 2018.

[20] J. Fang, H. Li, Y. Tang, and F. Blaabjerg, "Distributed power system virtual inertia implemented by grid-connected power converters," IEEE Transactions on Power Electronics, vol. 33, no. 10, pp. 8488-8499, Oct. 2018.

[21] J. Liu, Y. Miura, H. Bevrani, and T. Ise, "Enhanced Virtual Synchronous Generator Control for Parallel Inverters in Microgrids," IEEE Transactions on Smart Grid, vol. PP, no. 99, pp. 1-10, 2016.

[22] R. Rosso, S. Engelken, and M. Liserre, "Robust stability analysis of synchronverters operating in parallel," IEEE Transactions on Power Electronics, vol. 34, no. 11, pp. 11309-11319, Nov. 2019.

[23] P. Kundur, N. J. Balu, and M. G. Lauby, Power system stability and control. McGraw-Hill New York, 1994, vol. 7.

[24] M. A. Rahmani, Y. Herriot, S. L. Sanjuan, and L. Dorbais, "Virtual synchronous generators for microgrid stabilization : Modeling, implementation and experimental validation on a microgrid laboratory," in Asian Conference on Energy, Power and Transportation Electrification (ACEPT), vol. 2017-Decem. IEEE, Oct. 2017, pp. 1-8.

[25] A. Pearson, "Linear optimal control systems," IEEE Transactions on Automatic Control, vol. 19, no. 5, pp. 631-632, Oct. 1974.

[26] Y. Hirase, K. Sugimoto, K. Sakimoto, and T. Ise, "Analysis of Resonance in Microgrids and Effects of System Frequency Stabilization Using a Virtual Synchronous Generator,' IEEE Journal of Emerging and Selected Topics in Power Electronics, vol. 4, no. 4, pp. 1287-1298, Dec. 2016. 